\title{
Centrosymmetric crystal structure of racemic Z-DNA at ultrahigh resolution
}

\author{
Miroslaw Gilski $^{1,2}$, Pawel Drozdzal ${ }^{1}$, Mariusz Jaskolski ${ }^{1,2}$ \\ ${ }^{1}$ Department of Crystallography, Faculty of Chemistry, A. Mickiewicz University, Umultowska 89b, 61-614 \\ Poznan, Poland; E-mail: mirek@amu.edu.pl \\ ${ }^{2}$ Center for Biocrystallographic Research, Institute of Bioorganic Chemistry, Polish Academy of Sciences, \\ Noskowskiego 12/14, 61-704 Poznan, Poland
}

The self-complementary d(CGCGCG) hexanucleotide was synthesized as a racemic mixture and crystallized, producing a new crystal form of Z-DNA with $C 2 / c$ symmetry that diffracted X-rays to ultrahigh resolution $(0.78 \AA$ ). The structure was solved by several methods (direct methods, dual space and molecular replacement) and refined to an $R$-factor of $13.86 \%$. The asymmetric unit contains one ZDNA duplex and three magnesium sites. The crystal structure consist of both left-handed (2'deoxyribose D-form) and right-handed (L-form) Z-DNA duplexes, and exhibits the highest degree of disorder among all high-resolution structures of Z-DNA described so far. Specifically, nine out of the ten phosphate groups, 11 sugar moieties and also four nucleobases were modeled in alternative conformations. The crystal packing shows an unusual arrangement of the DNA helices in the unit cell, with end-to-end base-pair-stacked pseudohelical columns running along two different directions, and with consecutive hexameric duplexes tilted in the same direction with respect to the column axis. The hydrated $\mathrm{Mg}^{2+}$ ions play a significant role in the stabilization of the packing of the disordered $\mathrm{D}$ - and Lduplexes. 